\title{
DNN Based Plant Diseases Recognition Using Classification of Leaf Images
}

\author{
Rajaa J. Khanjar \\ Assist. Lecturer, Department of computer science, Baghdad University, Iraq
}

\begin{abstract}
Throughout the area of image processing, the new generation of convolutional neural networks CNNs has produced remarkable performances. The paper reflects on a new approach to the production of utilizing deep convolutionary networks of a model of the identification of plants centered on the picture classification of the surface. Throughout reality, a modern model of teaching and technique allows it simple and fast to introduce the program. With the ability to differentiate the plant leaves from the environment, the engineered model will recognise 13 specific forms of plant diseases from safe leaves. This approach for identifying plant disease was introduced for the first time, according to our understanding. The entire paper outlines all important measures possible for the introduction of this model for disease identification, beginning with the picture collection to establish a database reviewed by agricultural experts. The comprehensive CNN preparation was carried out by Caffe, a fundamental research system developed by Berkley Vision and Learning Center. On average, the experimental results of the built model were 91 to 98 percent reliable for separate class research.
\end{abstract}

Keywords: DNN, CNN, Leaf Image, Plant Disease.

\section{Introduction}

Plant disease is a common threat to yield and quality of global agricultural production and bears responsibility for a significant portion of production costs. It is reported that the loss caused by plant disease accounts for at least a $10 \%$ reduction in global food production. Most of disease damage evaluation and treatment are done by farmers in the field with the guidance of plant pathologists. Incorrect diagnosis and pesticide usage are very common ${ }^{1}$. Therefore, the prevention and control of plant disease have always been widely discussed because plants are exposed to outer environment and are highly prone to diseases. Normally, the accurate and rapid diagnosis of disease plays an important role in controlling plant disease, since useful protection measures are often implemented after correct diagnosis ${ }^{2}$. Many recognition and diagnosis method have been proposed by following the pipelined procedures of image segmentation, feature extraction, and pattern recognition. Recognition method following the pipelined procedures have made some progress. But these method are subject to two issues. First, the accuracy of these method greatly depends on the extraction and selection of visible disease features.
Specifically, features of visible disease symptoms should be extracted accurately and proper features should be selected. Second, the method following the pipelined procedures are relatively complicated. The presence of noises is largely unavoidable in disease images captured under field conditions, such as uneven illumination and clutter field background. This can severely decrease the quality of the features and affect the results of recognition. Therefore, many efforts are spent on eliminating the noises in conventional method to achieve robust results ${ }^{1}$. The recent trend in the development of computer vision and the use of various machine learning algorithms for plant disease classification has shown promising results in few selected diseases and crops. Evolution of deep Convolutional Neural Network (CNN) based architectures has further enhanced the accuracy of classification significantly ${ }^{3}$.

Literature Review: In this paragraph, different schemes have been proposed in automatic recognition of plant diseases as shown below:

An automatic identification of plant disease utilizing the capability of the digital image-based algorithm. This 
algorithm is proposed to cope with various diseases and it is easy to retrain it to include new diseases. Its histogram based structure enables it to be reasonably robust under different circumstances to capture images. The obtained results show that there is still a place for improvement. Many factors such as a considerable number of present disorders, heterogeneity of symptoms associated with the same disease and symptom similarities between different disorders may need the adoption of hybrid techniques combining expert systems, image processing, and other information gathering techniques may be the best hope to beat at least some of the disadvantages present in practice have been presented ${ }^{5}$.

System generative models are used for text and faces combined with standard shaded and textured regions models. This method makes it possible to interact and collaborate with these various versions to classify the images input. This device allows objects to be segmented, faces detected, and text detected and read in urban scenes. This system showed many cases where shaded models helped discover the face and text by explaining the shadows and absence sun glasses. The face and text models, in turn, improved segmentation quality. The present limits of this methodology lie in the standard class of objects that we are presently modelling. This restriction was inspired by the purpose of our software to identify the text and faces of visually disabled persons. But, in principle, this approach can include broad types of objects that have been presented ${ }^{6}$.

The method of detection depended on an analysing of the number of points clusters in combination with $\mathrm{CNN}$ as the final classification. The suggested method of evaluating clusters of dots depends on a combination of modelled fuzzy logic and graphics processing. The suggested detection and classification architecture has been tested and compared to other method in this field to demonstrate performance and to draw conclusions for further progress. The detection results combined with $\mathrm{CNN}$ enabled us to create a good classification of objects. In the case of face detection utilizing the large database, we attained the rate of about $83 \%$, while in the case of other items such as people about $75 \%$, animals about $55 \%$, and cars about $80 \%$. The performance showed the high potential of the technique proposed. correctly estimate the likelihood of ARX differentials in order to overcome these problems that have been presented ${ }^{7}$.

A support vector machine is used to identify the object of the selected image. The suggested approach of object detection is compatible with KPCA feature vector reduction and detection utilizing the SVM classification system. The extraction method of the feature from the image's global descriptors. General features are extracted and formed as a feature vector during the process of extracting an image. The vector parameter is developed for full-image training and utilizes KPCA to minimize dimensions. The SVM classifier is equipped using a reduced feature vector. Later test images are provided as input and the Classifier's output is checked. Back Propagation Neural Network is used for object recognition to show the validity of the SVM Classifier. SVM classifier outperforms from the comparison have been presented $^{8}$.

\section{The Proposed Method}

The framework of the proposed system comprises two main phases: a training phase and a classification phase. These two primary phases share the fundamental modules of the system (pre-processing module and features extraction module), as shown in Figure (1).
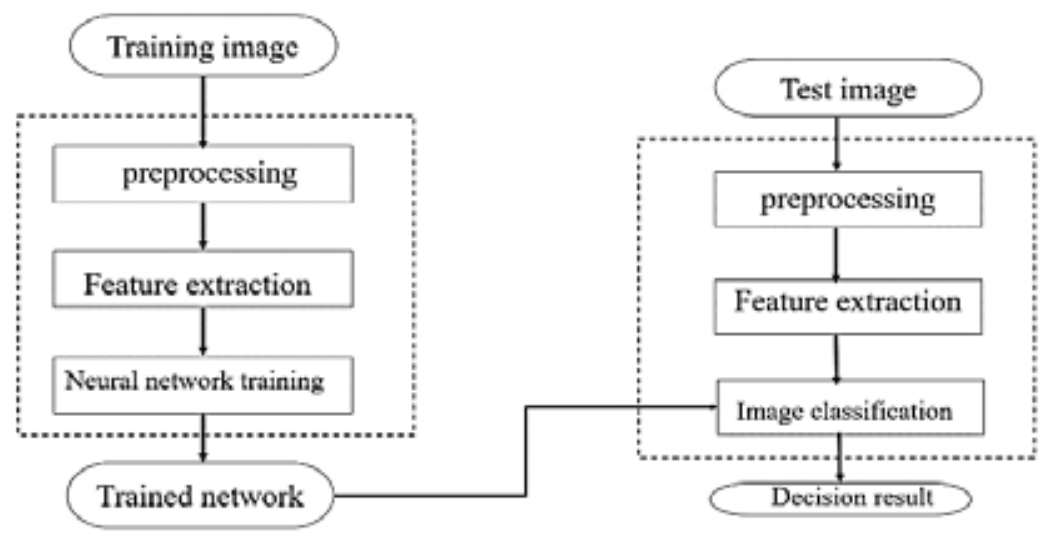

Figure (1): The framework of the proposed plant leaf diseases classification 
In the training phase, after the pre-processing and feature extraction steps extracted features vector, for each training sample, the features vectors are saved in the system database. While in the classification phase the system should match these feature vector of input plant leaf image with all vectors listed in the database and return the file name in dataset classifier. Matching and decision steps in the proposed system have been done by using a convolutional neural network (CNN).

System using Deep Learning Method: Deep learning neural network plant leaf diseases by image classification method is consist of two main stages:
1. Enrolment Phase: In this stage, many steps will be done such as pre-processing, localization, extracted features, (and labelled objects).

2. Matching Phase: In this stage, many steps will be used such as pre-processing, localization, extracted features, and matching process. Figure (2) explained the main stages of the proposed system are work and Figure (3) explained the main stages of $\mathrm{CNN}$ are work.

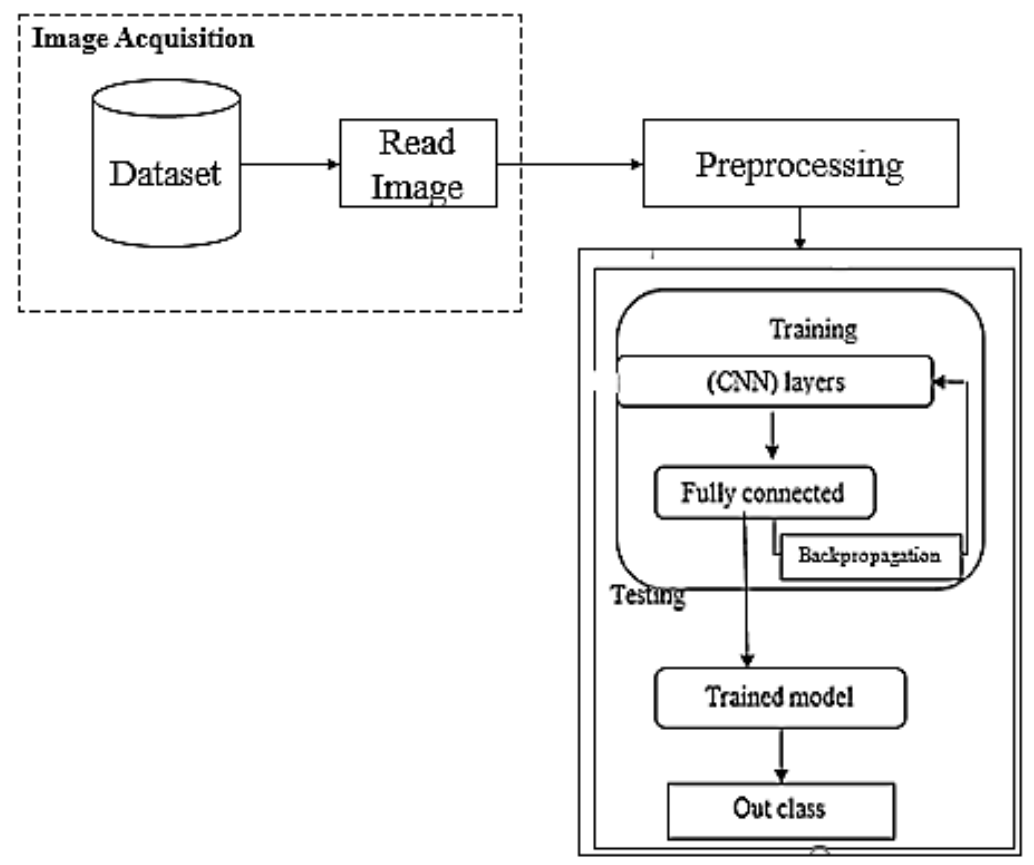

Figure (2): The main stages of the proposed system

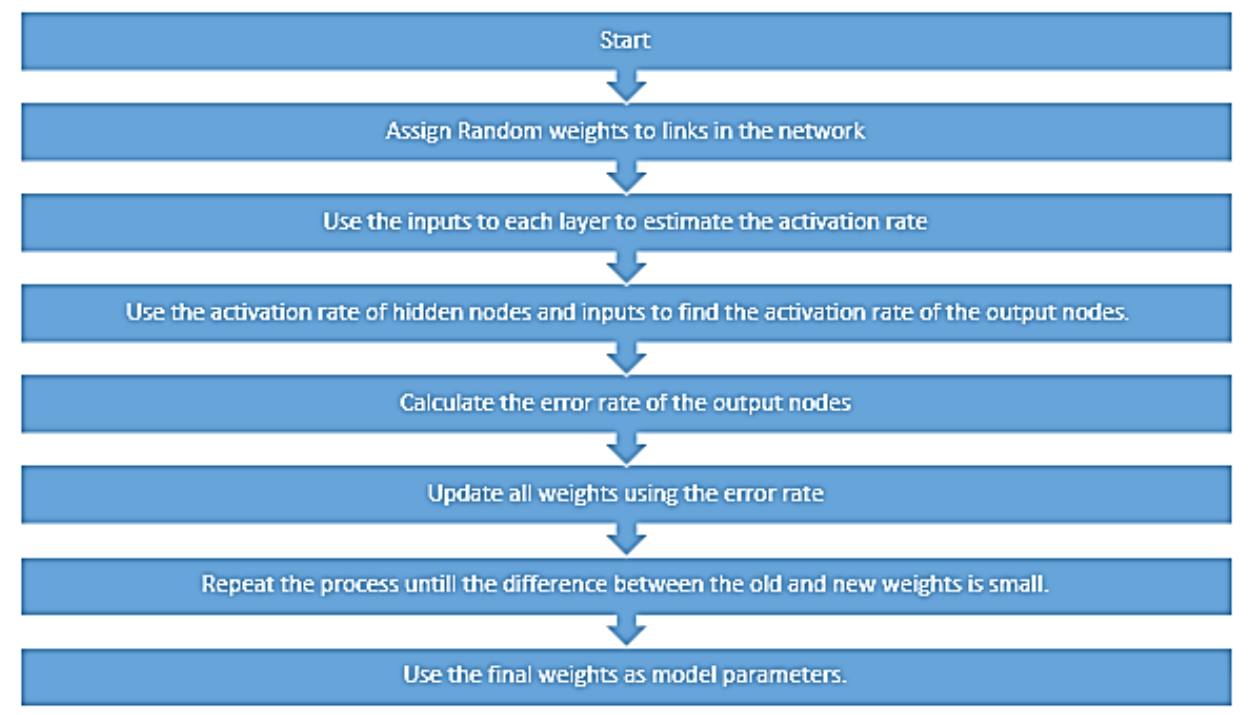

Figure (3): The main stages of $\mathrm{CNN}$ 
Image Acquisition: Acquisition of the image is always where vision systems begin in order to complete their set task. Once obtained, there are several different processing method which are able to be used in order to carry out several tasks in regards to the image. The reason why the image acquisition is always the first step in the workflow sequence is that if there is no image, processing is impossible. There are multiple method to acquire images including but not limited to, the use of cameras or scanners. The image which has been acquired needs to retain all features.
A. Standard Dataset: The images for 6 different diseases and healthy samples of pepper crops were obtained from the Plant Village dataset. The resolution of these images is $256 \times 256$ pixels and all these images are available in jpeg format. Figure (4) is showing the sample of Standard Dataset (Plant Village Database).

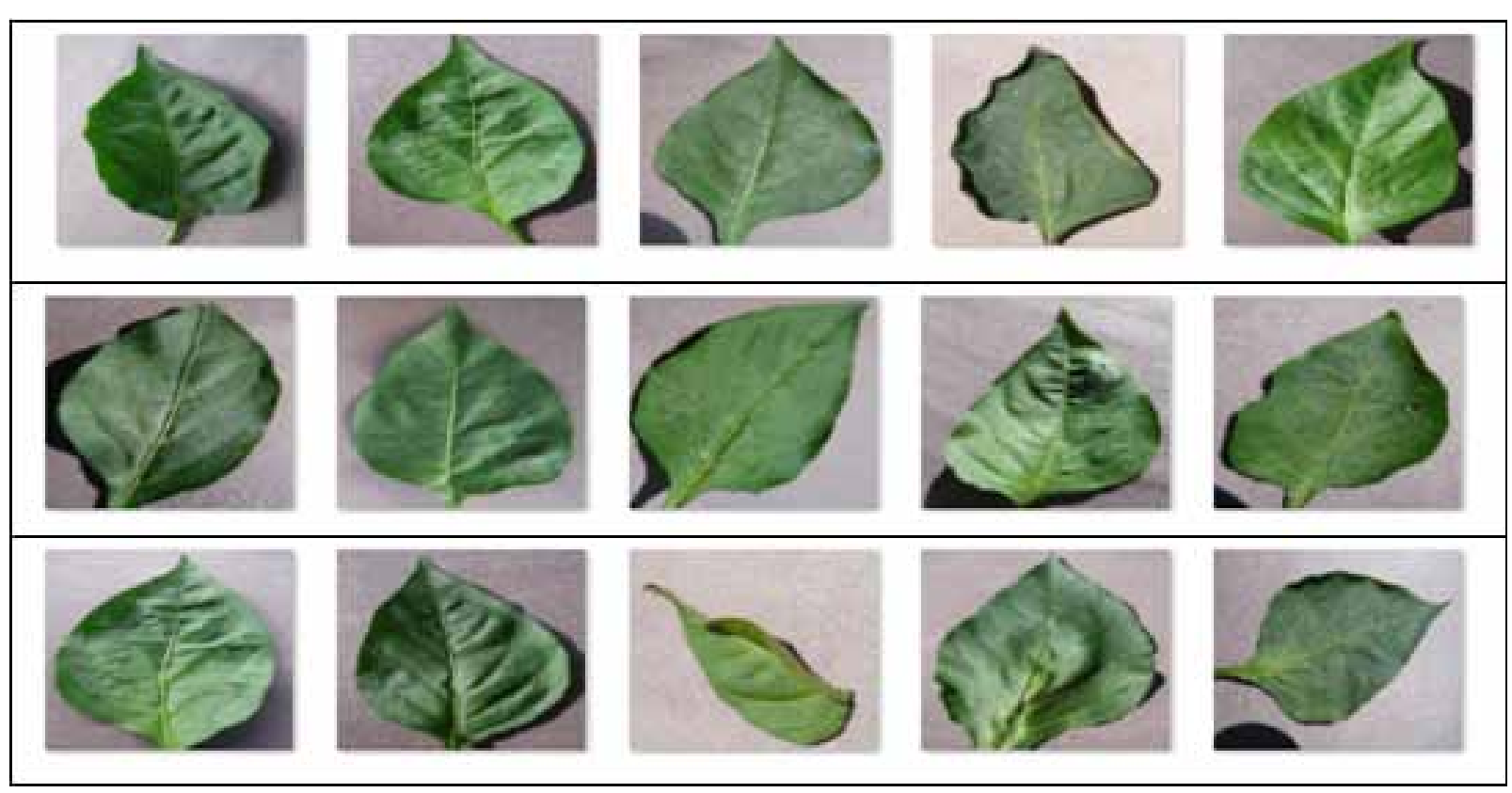

Figure (4) The sample of the Plant Village dataset

B. Enrolment Phase: At this stage all enrolled features are passed to the system at the same time in order to train the system. The enrolment phase is consisting of other sub steps:

I. Preprocessing Image: The first step in this work is applied pre-processing image to improve the plant leaf images. This technique is used to improve the image for the purpose of showing the image detail well because sometimes the images may be taken in conditions that are inappropriate in terms of light, noise or the size of the image is very large and does not produce good results.

A. Resize Image for Plant Leaf Diseases: Since the system aims to get a better style and the advantage of the picture of plant diseases will change the size of the image in a manner appropriate to the system. Because artificial neural network inputs are of a certain size, depending on the structure used in the network, the dimensions of the image are adjusted to fit the desired size. To achieve these dimensions, the method is applied to the image so that no information is lost from the image during the resizing process.

II. Feature Extraction: The most important stage in the identification system is feature extraction from samples such as plant leaf diseases. In the main proposed, the system uses the convolutional neural network $(\mathrm{CNN})$ in which the convolutional layer is in the role of the feature extraction stage. Figure (5) shown architecture of CNN that employed and explain each layer in details: 


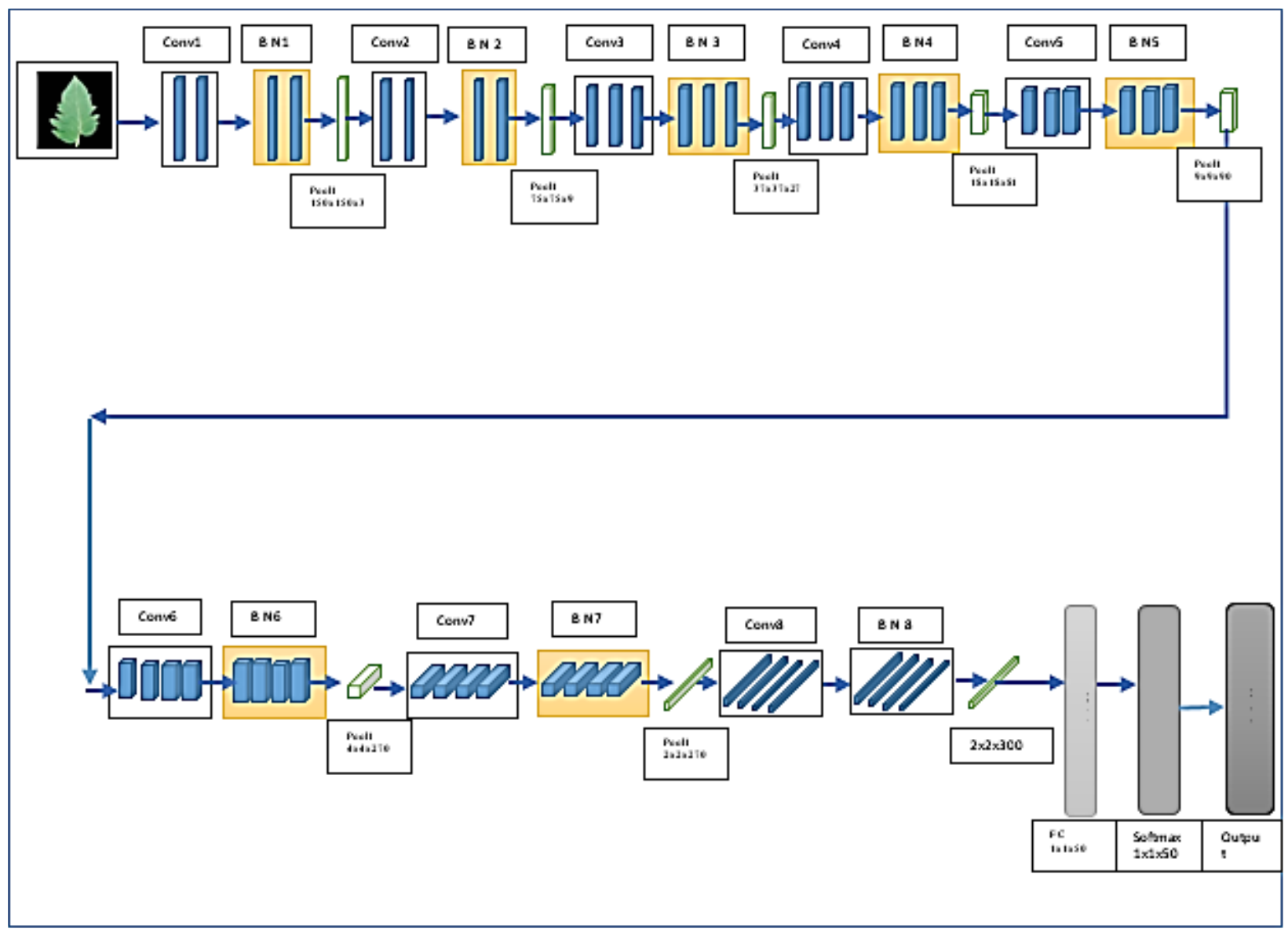

Figure (5): Architecture of Convolutional neural network

1. Convolutional Layer: Feature extraction layer, it operated by moving mask of weight slide over the original image and dot product multiplication is performed, to produce feature map. Weight is generated randomly, along with many iterations passes through BN, Pooling, RleU Layers, the Weight changes until reaching the best weight for this image, this weight represents the feature.

2. Batch Normalization Layer (BN): This layer is used to increase the speed of the training process and eliminate sensitivity to network initialization, it minimizes a large number of each channel, firstly, the activation of each channel is normalized by subtracting mean of mini batch and divide on standard deviation of mini batch, after that, the layer input is shifted by offset $\beta$, and then scale by factor $\gamma$. This layer is used between the convolutional layer and RleU layer.

3. Max Pooling Layer: In order to get rid of excess and unwanted feature, max pooling layer has been employed, so it returns an important feature, it does that by sliding mask with known dimension over feature map that results from previous convolutional layer, but the max is empty, at each stride, the result is the highest value lie under this mask.

4. RleU Layer: Since the images are naturally nonlinear, and contain non-linear features such as color and border, the rectifier function is applied to increase non-linearity of the image, i.e. RleU layer is used to ensure only robust feature by taking only positive numbers and convert all negative numbers to zero.

5. Fully Connected Layer: It's represent feature vector that hold the most important information for the input, it is gather the feature from all previous convolutional layer during training operation that can be used for classification later. i.e. train hidden layer to give probability of each class.

6. Softmax Layer: The output of Softmax layer is probability number ranging between zero and one (0-1), each class has its probability, for example 
$(0.011,0.005)$, give high probability to the candidate class and decrease other classes.

7. Loss Function Layer: The loss function is used to determine the loss (error) at each trading epoch, it is also an important factor that the update of weight during backpropagation depends on i.e. it shown the difference between predicted output and true label.

C. Matching and Decision Stage: To find the difference between objects coordinate is used Euclidian distance. therefore, determine the decision of the identification system used Matching operation based on Euclidian distance value. each class has its upper and lower limitations when the result of distance lies between the limitations of any class, this means the examined sample belongs to this class.

4. Experimental Results: This method of detecting objects works best with objects that display unrepeated patterns of texture, resulting in special matches of features. With uniformly color objects or items containing repeating patterns, this method is unlikely to work well.

a. Pre-processing stage: This is the important stage during which the original image is converted to a grayscale image and ROI is extracted as shown in Figures (6).

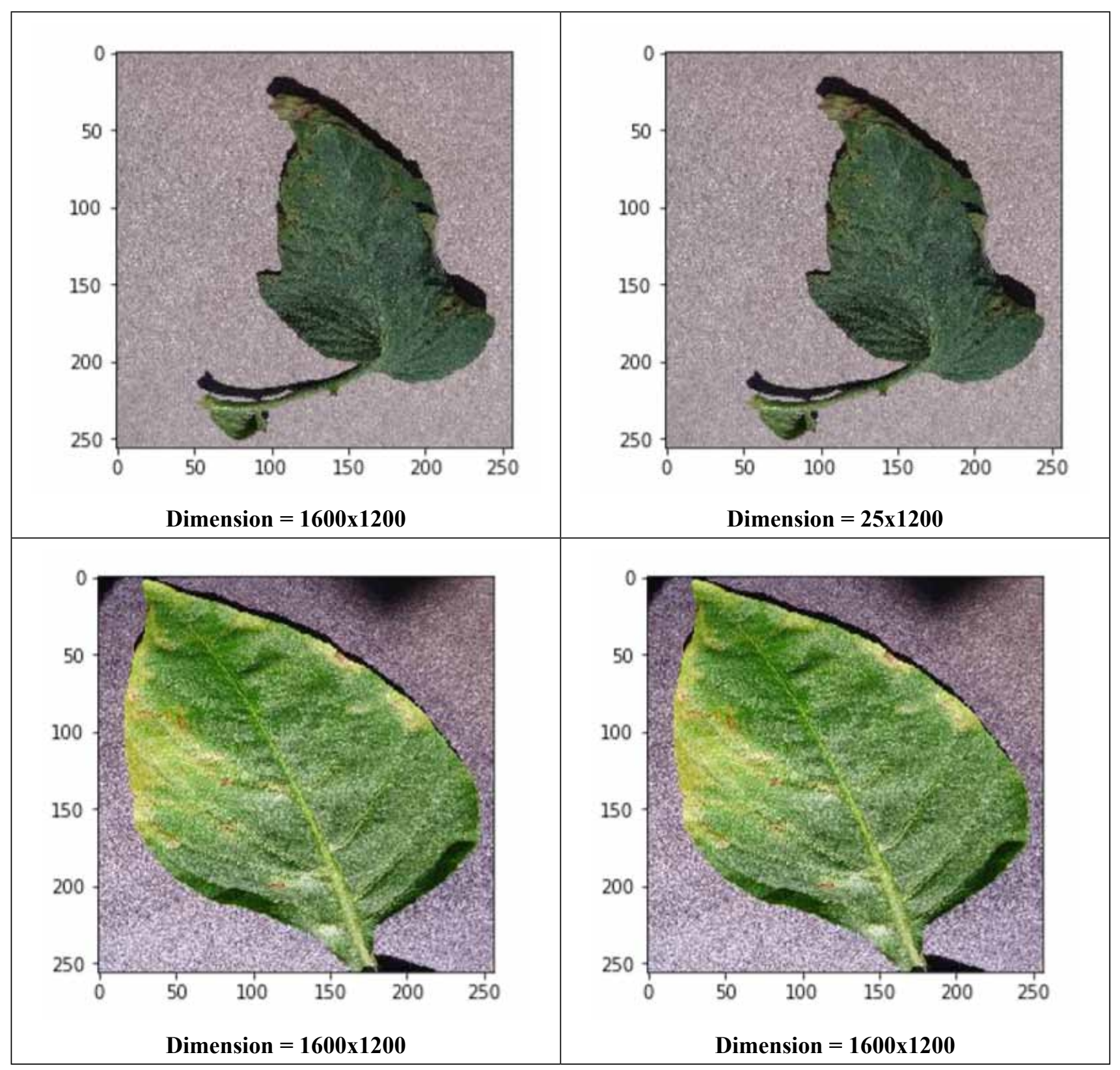




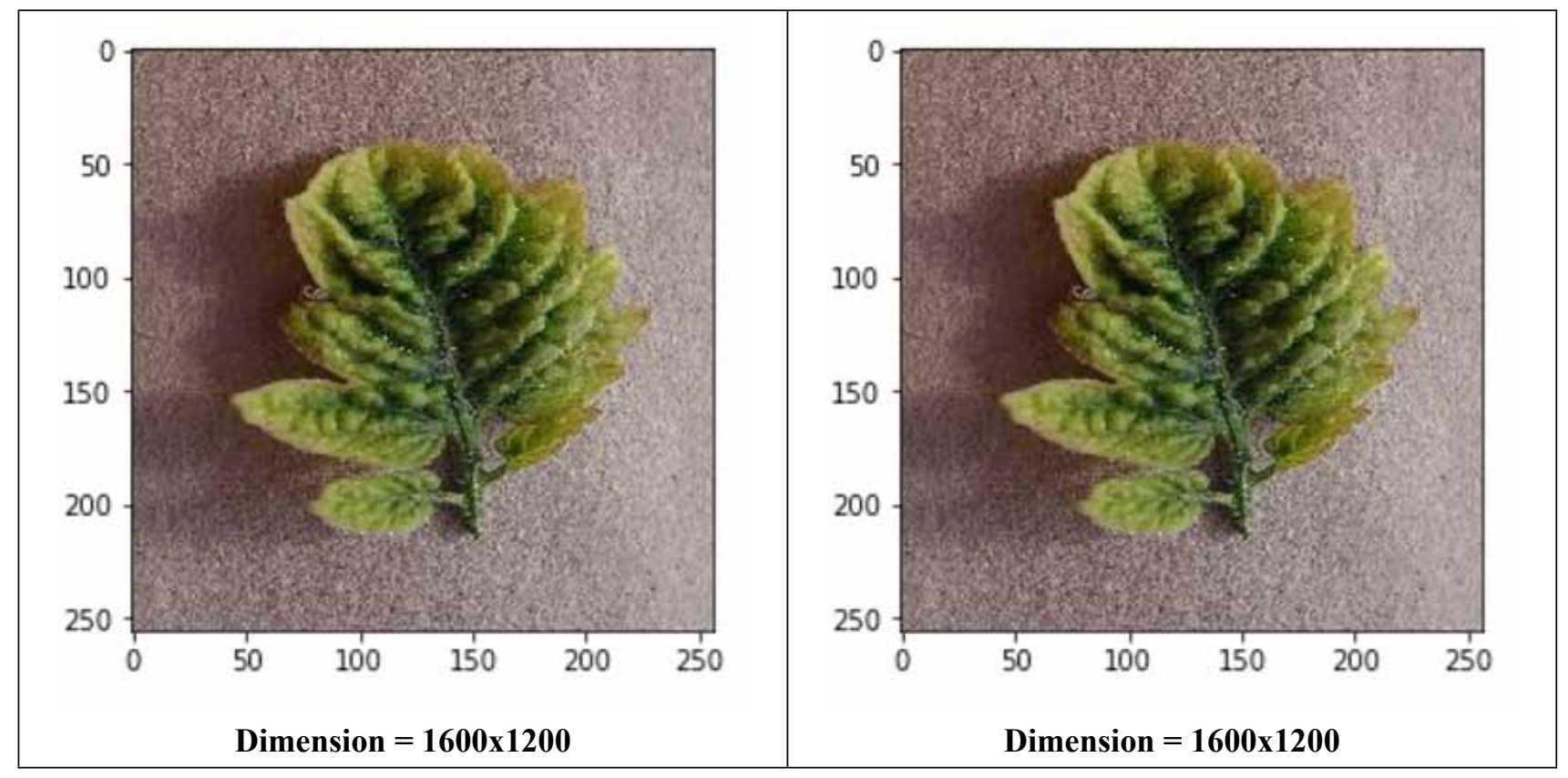

Figure (6): Resize Image

b. Convolutional neural network (CNN): Feature extraction is the essence (core) of the detection Plant Diseases Leaf system, convolutional layer (conv_) in CNN is feature extraction layer, it works by sliding the weight mask over the input image and perform dot product multiplication, the result is called feature map, feature map of first convolutional layer(conv_1) act as input to Batch Normalization Layer (batchnorm_1) which minimize the numbers of feature map (numbers that represent input image after convolutional layer 1) to speed up training process, the output is going forward to first maxpooling layer, that takes only an important feature (highest values) and ignores the other, to increase the difference between individuals feature. Figure shows the output of each convolutional layer on $\mathrm{CNN}$ next layer is RleU that ignore the negative number to ensure only vital feature are remain, so the size of the feature map is minimized.

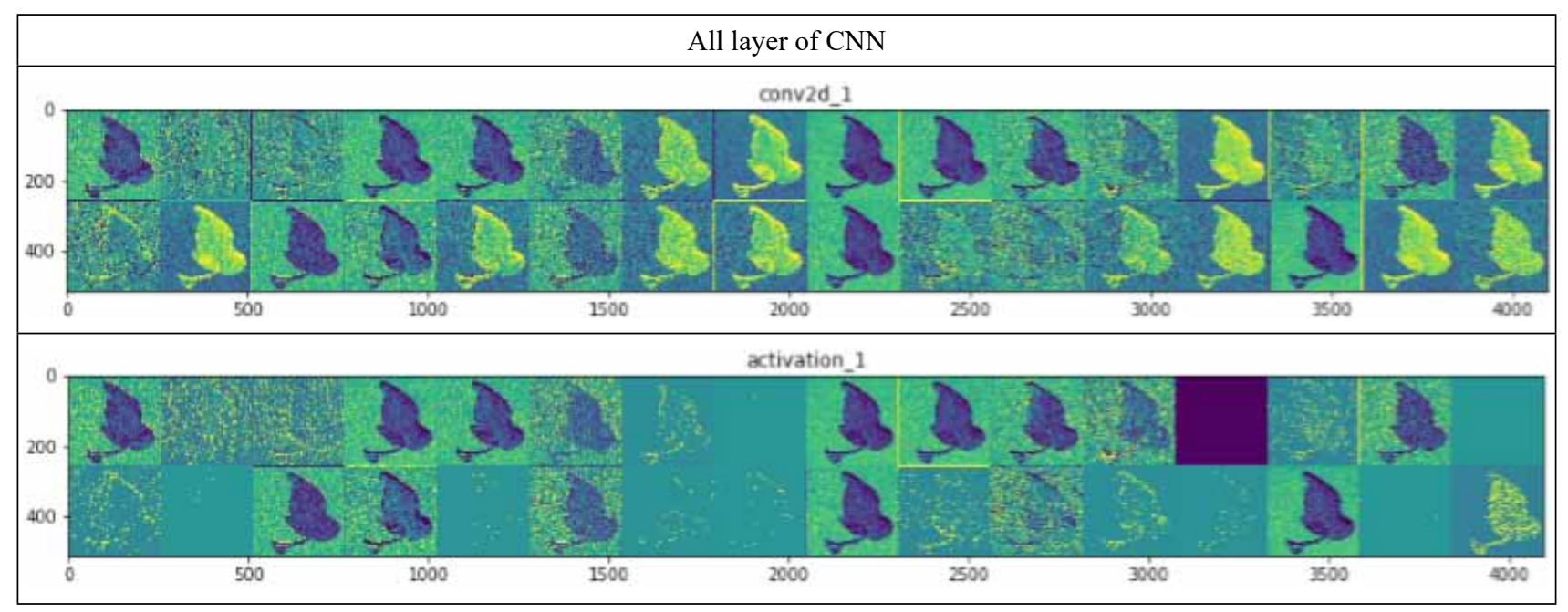



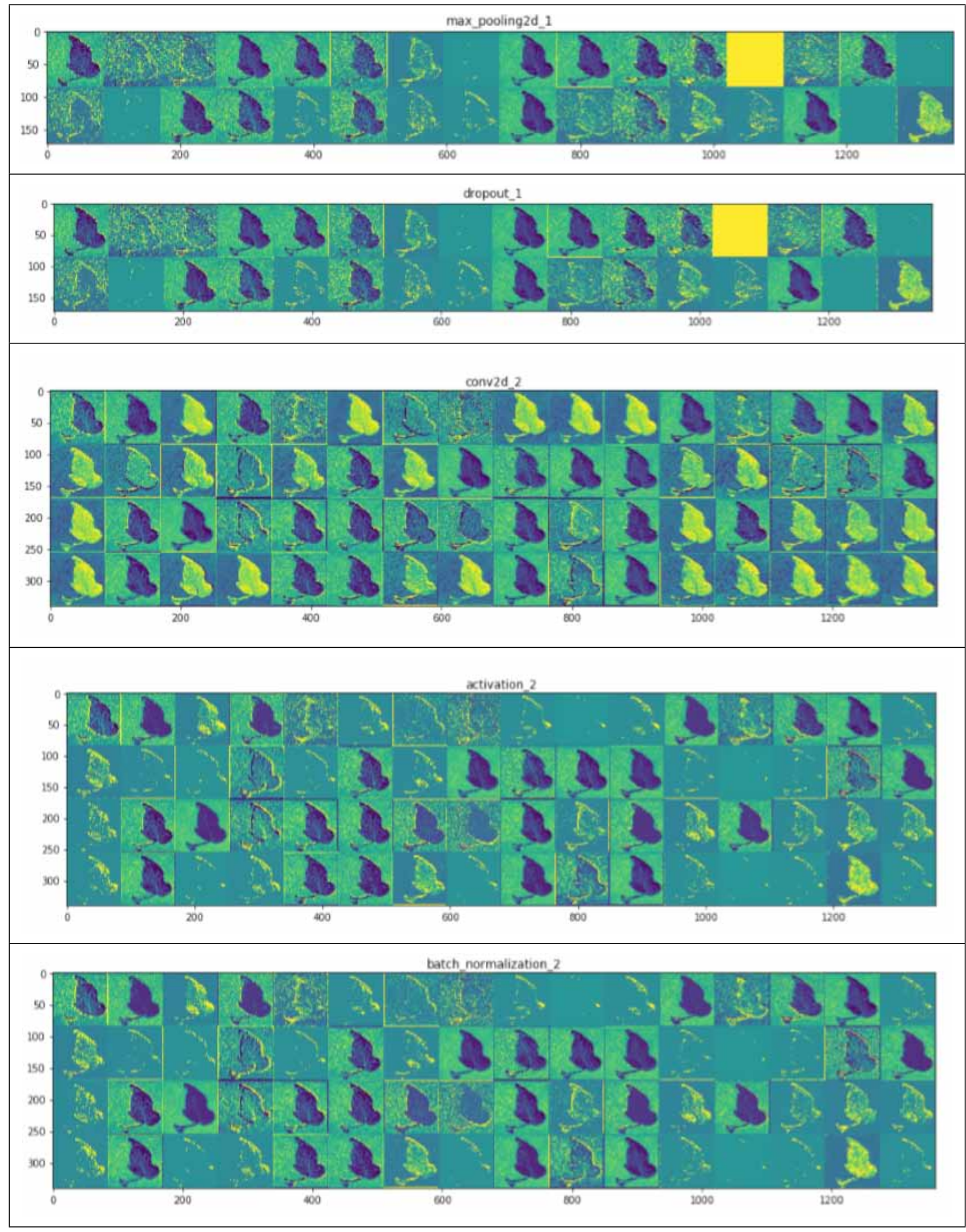


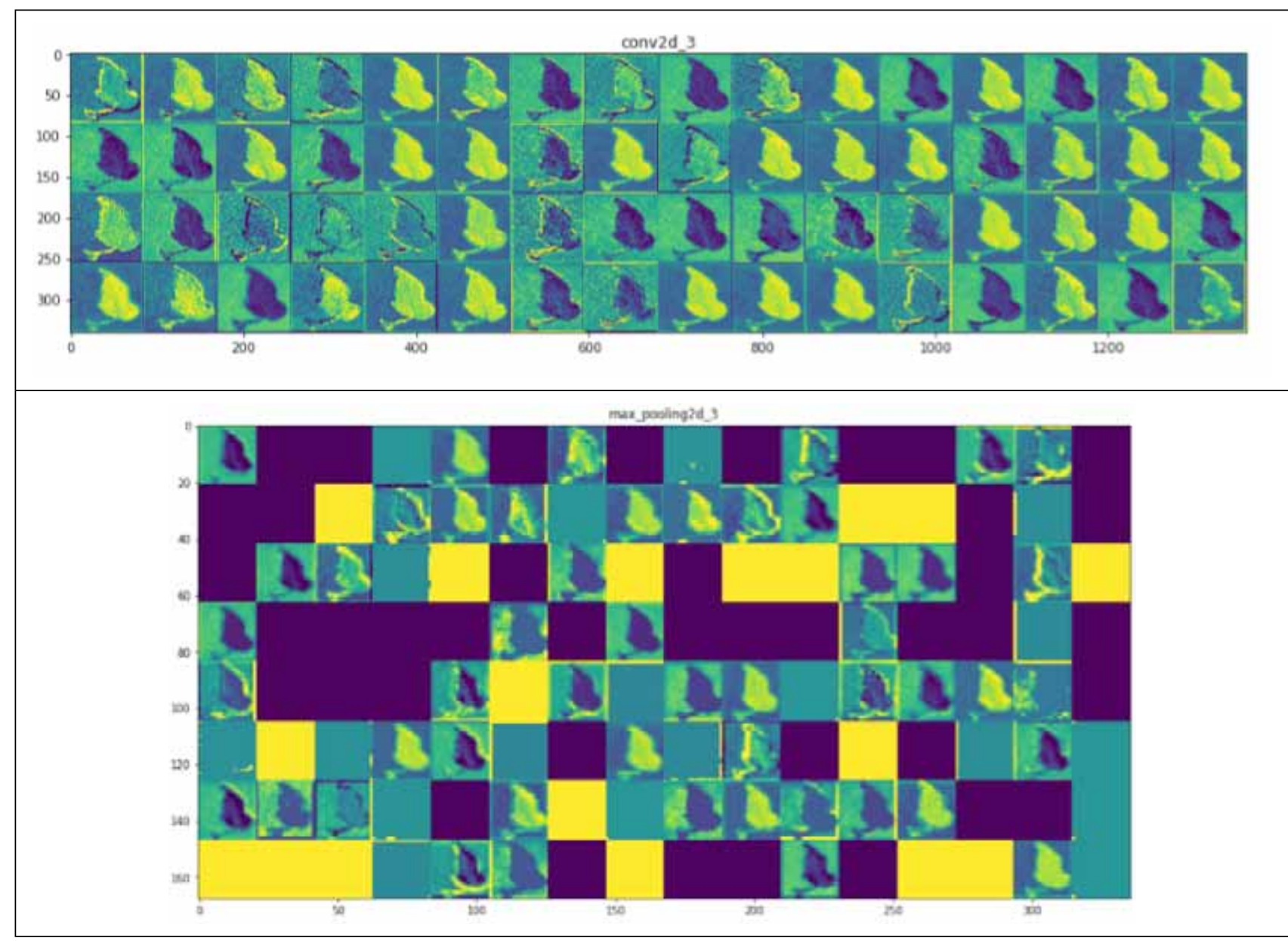

Figure (7): The output of each convolutional layer on CNN

\section{Conclusion}

In this paper, the goal is to automatically identify and detect herbal diseases from leaf images via a new approach of deep education process. The model was established to identify the involvement of leaves and differentiate between stable leaves and 13 diseases, which can be visually identified. The full method was defined from the set of images used for testing and evaluation, the pre-processing and extension of images and finally the deep CNN testing and fine-tuning technique. Numerous experiments have been performed to verify the newly developed model 's efficiency. New picture archive of plants disease was developed with over 3,000 initial photographs taken from Internet sources accessible and expanded by correct transformations to more than 30,000. Regarding specific class checks, the experimental findings obtained accuracy between $91 \%$ and $98 \%$. The average finish of the trainer qualified was $96.3 \%$. Fine-tuning revealed no major improvements in average precision; however, the cycle of improvement had a stronger effect on the performance.

Financial Disclosure: There is no financial disclosure.

Conflict of Interest: None to declare.

Ethical Clearance: All experimental protocols were approved under the Department of computer science and all experiments were carried out in accordance with approved guidelines.

\section{References}

1. KA Garrett, SP Dendy, EE Frank, MN Rouse, SE Travers. Climate change effects on plant disease: genomes to ecosystems," Annual Review of Phytopathology. 2006; 44: 489-509.

2. SMCoakley, H Scherm, S Chakraborty. Climate change and plant disease management," Annual 
Review of Phytopathology. 1999; 37: 399-426.

3. S Chakraborty, AV Tiedemann, PS Teng. Climate change: potential impact on plant diseases," Environmental Pollution. 2000; 108: 317-326.

4. AJ Tatem, DJ Rogers, SI Hay. Global transport networks and infectious disease spread," Advances in Parasitology. 2006; 62: 293-343.

5. JR Rohr, TR Raffel, JM Romansic, H McCallum, PJ Hudson. Evaluating the links between climate, disease spread, and amphibian declines," Proceedings of the National Academy of Sciences of the United States of America. 2008; 105: 1743617441.
6. T Van der Z. Present worldwide distribution of fire blight," in Proceedings of the 9th International Workshop on Fire Blight, vol. 590, Napier, New Zealand, October 2001.

7. SA Miller, F D Beed, CL Harmon. Plant disease diagnostic capabilities and networks," Annual Review of Phytopathology, vol. 47, pp. 15-38, 2009.

8. MB Riley, MR Williamson, O Maloy. Plant disease diagnosis. The Plant Health Instructor,” 2002. 